\title{
Effects of Initiator on the Morphology and Compatibility of Components of Poly(methyl methacrylate)/Polystyrene Composite Latex and Polymer Blends
}

\author{
Chia-Fen LeE \\ Department of Cosmetic Science, Chia Nan College of \\ Pharmacy and Science, Tainan, Taiwan, R.O.C.
}

(Received December 1, 1999)

\begin{abstract}
In this study, the poly(methyl methacrylate)/ polystyrene (PMMA/PS) composite latex was synthesized by soapless seeded emulsion polymerization in two steps. In the first step reaction, potassium persulfate $\left(\mathrm{K}_{2} \mathrm{~S}_{2} \mathrm{O}_{8}\right)$ was used as initiator to synthesize the PMMA seed latex. In the second step reaction, potassium persulfate, 4,4'-azobis(4cyanovaleric acid) (ABCVA) and azobis(isobutyronitrile) (AIBN) was used as initiator respectively to synthesize polystyrene in the presence of PMMA seed latex to form three PMMA/PS composite latexes with different morphology. The morphology of the PMMA/PS composite polymer latex could be controlled by the initiator in the second step reaction. The three composite polymer latexes were analyzed by TLC-FID. The results showed that there were PMMA-graft-PS copolymer consisted in the PMMA/PS composite polymer latex. The amount of PMMA-graft-PS copolymer was influenced by the initiator used in the second stage reaction. The compatibility of PMMA phase and PS phase in the composite polymer latex was influenced by the amount of PMMA-graft-PS copolymer. The effects of thermal annealing on the morphology of composite polymer latex were investigated. PMMA/PS composite polymer latex was blended with commercial PMMA and commercial PS to form the commercial polymer/composite latex polymer blends. The effects of thermal annealing on the morphology of polymer blends were studied. The results showed that the PMMA/PS composite polymer latex had the ability to stabilize the morphology of the polymer blend during thermal annealing.

KEY WORDS Morphology / Composite Latex/Polymer Blends / Soapless Seeded Emulsion Polymerization /
\end{abstract}

The seeded emulsion polymerization is often used to synthesize polymer/polymer composites in industry. There are many factors to control the kinetics and morphology of polymer, such as monomer added to the system, ${ }^{1,2}$ sequence of monomers fed to the system, monomer ratio in two stages, the hydrophilicity of the monomers, surface tension, molecular weights of the polymers, ${ }^{2-4}$ compatibility of the polymers, ${ }^{5}$ and properties of the initiators. ${ }^{6}$

Okubo et al. pointed out that the morphology of polymer particles changes with the viscosity of polymerization loci, and hydrophilicity of the two polymers. ${ }^{7-9}$ Muroi and Hosoi ${ }^{10}$ studied the structures of core-shell latex particles. They pointed out that the polymer particle formed an inverted core-shell morphology when the hydrophobic monomer of the second stage was polymerized in the presence of hydrophilic seeds. In our previous work, the kinetics of soapless seeded emulsion polymerization were studied. ${ }^{11-14}$ The morphology, thermal properties and mechanical properties of poly(butyl acrylate)/ polystyrene (PBA/PS) composite latex particles were investigated. ${ }^{15}$ We found that the morphology of PBA/PS composite latex was core-shell structure. A thin layer of PBA-graft-PS copolymer was formed on the boundary of the core region and shell region. The PBA-graft-PS copolymer increased the degree of compatibility of the PBA and PS phases. Moreover, we also investigated ${ }^{16}$ the effects of composite polymer latex on the morphology and mechanical properties of polymer blends. We found that the composite polymer latex stabilized the morphology of polymer blend under the effect of thermal annealing. The effect of thermal annealing decreased the tensile strength of the conventional polymer blend but not the tensile strength of the polymer blend that contained composite polymer latex. In recent years, although there are articles that investigate the effects of initiators on the morphology of composite polymer latex, there are no studies on the initiator effects on the compatibility of the components of composite polymer latex. In this investigation, the morphology of the composite latex that was synthesized using different initiators was investigated. The effect of the composite latex on the compatibility of the component of commercial polymer/composite polymer latex blends was investigated.

\section{EXPERIMENTAL}

\section{Material}

Methyl methacrylate and styrene were distilled under nitrogen atmosphere and reduced pressure prior to polymerization. Water was redistill and deionize. Other chemicals were of analytical grade and used without further purification.

\section{Polymerization}

Synthesis of PMMA Seed Latex. In the first stage reaction, PMMA seed latex was synthesized via soapless emulsion polymerization. The reaction was carried out at $80^{\circ} \mathrm{C}$ for $1 \mathrm{~h}$. Stirring was controlled at $300 \mathrm{rpm}$. After polymerization of the first stage, the PMMA latex was used as seeds to proceed the second stage reaction.

Synthesis of PMMA/PS Composite Polymer Latex. In the second stage, quantitative styrene monomer was poured into the PMMA seed emulsion, and stirred to cause swelling, and heated in a water bath to $70^{\circ} \mathrm{C}$. Aqueous solution of $\mathrm{K}_{2} \mathrm{~S}_{2} \mathrm{O}_{8}$ or 4,4-azobis(4-cyanovalerica cid) (ABCVA) was poured into the reaction to start the second stage. After $2 \mathrm{~h}$, the PMMA/PS core-shell composite polymer latex with weight ratio of PMMA/PS of $1 / 2$ was produced. Azobis(isobutyronitrile) (AIBN) was dis- 
Table I. Weight average molecular weight of polymer latex and commercial polymer

\begin{tabular}{lc}
\hline Polymer latex & $\begin{array}{c}\text { Weight average molecular weight of } \\
\text { polymer latex }\end{array}$ \\
\hline PMMA seed & 423500 \\
MS-KPS & 425900 \\
MS-ABCVA & 422300 \\
MS-AIBN & 419900 \\
Commercial PMMA & 426500 \\
Commercial PS & 425000 \\
\hline
\end{tabular}

solved in the quantitative styrene monomer, and poured into the poly(methyl methacrylate)(PMMA) seed emulsion for swelling at room temperature. The reaction system was heated to the reaction temperature to begin the second stage reaction. After $2 \mathrm{~h}$, the PMMA/PS core-shell composite polymer latex with weight ratio of PMMA/PS of $1 / 2$ was produced. The PMMA/PS composite polymer latex synthesized using $\mathrm{K}_{2} \mathrm{~S}_{2} \mathrm{O}_{8}$ as initiator in the second stage reaction was symbolized as MS-KPS. The PMMA/ PS composite polymer latex synthesized using ABCVA as initiator in the second stage reaction was symbolized as MS-ABCVA. The PMMA/PS composite polymer latex synthesized using AIBN as initiator in the second stage reaction was symbolized as MS-AIBN. Weight average molecular weight of the PMMA/PS composite polymer latex is listed in Table I.

\section{Thin-Layer Chromatographic (TLC) Analysis}

Qualitative Analysis. Polymers obtained from PMMA/ PS composite latex, PMMA latex and PS latex were dissolved in tetrahydrofuran (THF) respectively to prepare polymer solution at concentration of $0.005 \mathrm{~g} \mathrm{~mL}$. One microliter of each polymer solution was taken out and dripped on TLC plates. The polymer components in the sample were separated with $\mathrm{CHCl}_{3}$ in front of the TLC plate. The sample was separated again with appropriate solvent $\mathrm{CHCl}_{3} / \mathrm{CH}_{3} \mathrm{COOC}_{2} \mathrm{H}_{5}$ (weight ratio of $\mathrm{CHCl}_{3} / \mathrm{CH}_{3}$ $\mathrm{COOC}_{2} \mathrm{H}_{5}$ is $60 / 40$ ) at the middle of the TLC plate. The TLC plates were sprayed with Thymol Blue in a water ethyl alcohol mixture. $\mathrm{H}_{2} \mathrm{SO}_{4}$ at $10 \mathrm{~N}$ was sprayed on the TLC plates. Finally, the TLC plates with polymers were dried in an oven.

Quantitative Analysis. Composite polymer latices were quantitatively analyzed by a thin-layer chromatographic/flame ionization detector (TLC-FID). One microliter of polymer solution of the sample in THF ( concentration was $0.005 \mathrm{~g} \mathrm{~mL}$ ) was dripped onto a thin quartz rod coated with a silica gel $75 \mu \mathrm{m}$ thick. The polymer components in the sample were separated with $\mathrm{CHCl}_{3}$ in front of the quartz rod, and then the sample was separated again with appropriate solvent, $\mathrm{CHCl}_{3} /$ $\mathrm{CH}_{3} \mathrm{COOC}_{2} \mathrm{H}_{5}$, at a weight ratio of $60 / 40$ at the middle of the quartz rod. The compositions of the samples on the rods were measured by FID.

Compatibility of Components of Composite Polymer Latex. For analysis of the compatibility of components of composite polymer latex, the glass transition temperature $\left(T_{\mathrm{g}}\right)$ of the polymer latex was measured using differential scanning calorimetry (DSC), at a heating rate of $10^{\circ} \mathrm{C} \min ^{-1}$. The composite polymer latex had two $T_{\mathrm{g}} \mathrm{s}$ : PMMA $\left(T_{\mathrm{g}_{\mathrm{PMMA}}}\right)$ and PS $\left(T_{\mathrm{gPS}_{\mathrm{PS}}}\right) . T_{\mathrm{g}}$ of the individual PMMA phase of the composite polymer latex was obtained by measuring the PMMA seed latex with DSC. To measure
$T_{\mathrm{g}}$ of the individual PS phase, the crosslinking PMMA polymer latex was used as seeds to synthesize crosslinking PMMA/linear PS composite polymer latex, which had the crosslinking PMMA and linear PS phases. The crosslinking PMMA/linear PS composite polymer latex was dissolved in solvent to form the polymer solution. Because the PS phase was linear, the solvent dissolved the linear PS phase, but not the crosslinking PMMA phase. The crosslinking PMMA and linear PS were separated by centrifugation. The linear PS polymer was dried by oven, and $T_{\mathrm{g}}$ of PS was measured by DSC. Compatibility of the components of PMMA/PS composite latex was defined as:

$$
\left|\Delta T_{\mathrm{g}}{ }^{0}-\Delta T_{\mathrm{g}}\right| / \Delta T_{\mathrm{go}}^{0}
$$

where $\Delta T_{\mathrm{g}}^{0}=\left|\Delta T_{\mathrm{g}_{\text {PMMA }}}-\Delta T_{\mathrm{g}}^{0}{ }_{\mathrm{PS}}\right|$

$$
\Delta T_{\mathrm{g}}=\mid \Delta T_{\mathrm{g}_{P M M A}}-\Delta T_{\mathrm{g}}
$$

$\Delta T_{\mathrm{g}_{\text {PMMA }}}^{0}: T_{\mathrm{g}}$ of individual PMMA phase

$\Delta T_{\mathrm{g} \text { FS }}^{0}: T_{\mathrm{g}}$ of individual PS phase

$\Delta T_{\mathrm{g}}: T_{\mathrm{g}}$ of PMMA phase of PMMA/PS composite polymer latex

$\Delta T_{\mathrm{g} \text { PS }}: T_{\mathrm{g}}$ of PS phase of PMMA/PS composite polymer latex

\section{Preparation of Polymer Blend}

Conventional Polymer Blend. PMMA and PS of commercial grade were blended (weight average molecular weights of PMMA and PS is 426500 and 425000 respectively, and weight ratio of PMMA/PS is $1 / 2$ ) by a twin screw extruder. The temperature for blending was $210^{\circ} \mathrm{C}-245^{\circ} \mathrm{C}$, and the speed of twin screw was $40 \mathrm{rpm}$.

Commercial Polymer/Composite Latex Polymer Blend. The PMMA/PS composite polymer latex (weight ratio of PMMA/PS is $1 / 2$ ) and commercial grade PMMA and PS (weight ratio of PMMA/PS is $1 / 2$ ) were blended by the twin screw extruder. The temperature for blending was $210^{\circ} \mathrm{C}-245^{\circ} \mathrm{C}$, and the speed of twin screw was $40 \mathrm{rpm}$. Weight percent of core-shell composite polymer latex was $30 \%$ and weight percent of commercial grade polymer was $70 \%$.

\section{Thermal Annealing}

At the constant temperature of $180^{\circ} \mathrm{C}$, the samples were annealed in the oven.

\section{Observation of Morphology}

The samples were ultramicrotomed to form sections $900 \AA$ thick, and stained with $\mathrm{RuO}_{4}$. A transmission electron microscope (TEM) (Model: Hitachi H-600) was used to observe stained sections.

\section{RESULTS AND DISCUSSION}

\section{Morphology of Composite Polymer Latex}

Figure 1a shows the TEM photograph of the morphology of PMMA/PS composite polymer latex synthesized using $\mathrm{K}_{2} \mathrm{~S}_{2} \mathrm{O}_{8}$ as initiator in the second stage, and PMMA seed latex swelled styrene monomer for $24 \mathrm{~h}$ before the second stage reaction. The morphology of PMMA/PS composite polymer latex showed a core-shell structure. The core region was PMMA and shell region was PS. The core--shell morphology was due to the initiator that used in the second stage, $\mathrm{K}_{2} \mathrm{~S}_{2} \mathrm{O}_{8}$, which was 

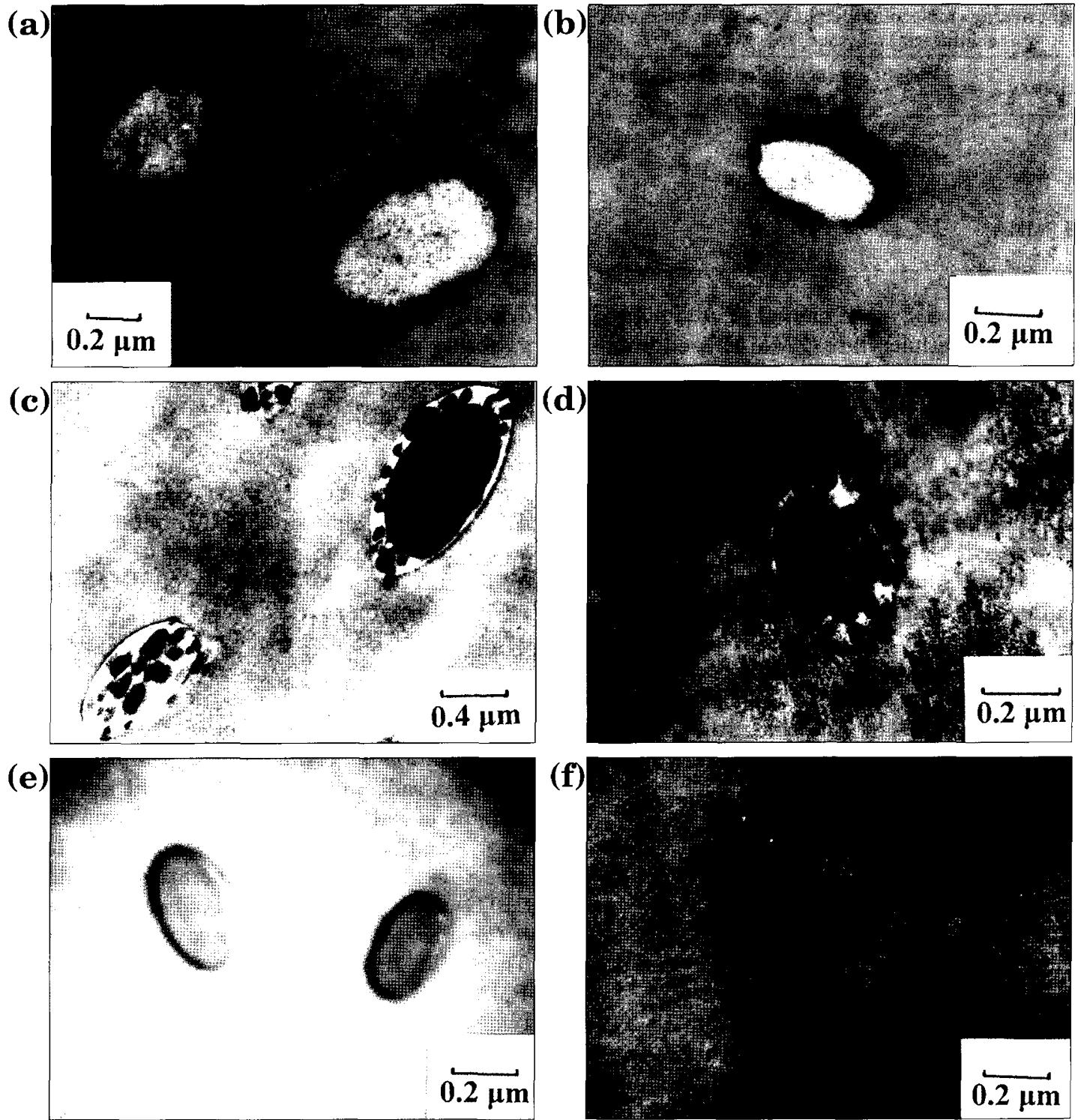

Figure 1. TEM photograph of sectioned polymer particles at the end of second-stage (dark zone is PS, bright zone is PMMA) . (a)Using $\mathrm{K}_{2} \mathrm{~S}_{2} \mathrm{O}_{8}$ as initiator in the second-stage styrene swelling $24 \mathrm{~h}$. (b)Using $\mathrm{K}_{2} \mathrm{~S}_{2} \mathrm{O}_{8}$ as initiator in the second-stage styrene swelling $0 \mathrm{~h}$. (c)Using ABCVA as initiator in the second-stage styrene swelling $24 \mathrm{~h}$. (d)Using ABCVA as initiator in the second-stage styrene swelling $0 \mathrm{~h}$. (e)Using AIBN as initiator in the second-stage styrene swelling $24 \mathrm{~h}$. (f)Using AIBN as initiator in the second-stage styrene swelling $0 \mathrm{~h}$.

water-soluble. The $\mathrm{SO}_{4}{ }^{-}$group decomposed from $\mathrm{K}_{2} \mathrm{~S}_{2} \mathrm{O}_{8}$, on the surface of the latex particle. Although the styrene was swollen into the inner region of PMMA seed latex, the styrene monomer diffused to the shell region from the inner region of PMMA seed region ${ }^{12,13}$ during polymerization. Polymerization proceeded on the shell region of the latex particle. The PMMA core was formed in the first stage reaction and then PS by formation of the PS shell in the second stage reaction. The morphology of the PMMA/PS composite polymer latex was thus a coreshell. Figure 1b shows a TEM photograph of the morphology of PMMA/PS composite polymer latex, synthesized using $\mathrm{K}_{2} \mathrm{~S}_{2} \mathrm{O}_{8}$ as initiator, and without PMMA seed latex swollen styrene monomer, before the second stage. The TEM photograph of Figure $1 \mathrm{~b}$ shows the morphology of core-shell structure. Without the swelling, the styrene monomer was polymerized directly in the presence of PMMA seed latex, the styrene monomer could not swell into the PMMA seed latex. During the second stage, the styrene monomer diffused to the surface of PMMA seed latex from styrene monomer drops in the emulsion system. Polymerization proceeded on the surface of PMMA seed latex to form the PS shell. The morphology of PMMA/PS composite polymer latex showed a core-shell structure. Figure 1c shows the TEM photograph of the morphology of PMMA/PS composite polymer latex, synthesized using ABCVA as initiator in the second stage and PMMA seeds swollen styrene monomer for $24 \mathrm{~h}$. The morphology had core and shell regions. The core region was the PS phase that was dispersed in the PMMA continuous phase. Since the initiator ABCVA was poorly water-soluble. So, during polymerization, ABCVA could not completely anchor to the surface of the polymer latex. Most of ABCVA diffused into the PMMA seed latex, and polymerization proceeded mostly in the inner region of PMMA seed latex. The morphology of the 


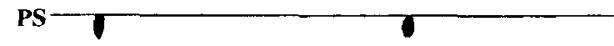

PMMA-PS copolymer

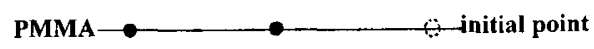

(a)

(b)

(c)

(a) PMMA/PS linear composite latex

(b) pure PMMA

(c) pure PS

Figure 2. TLC figures of PMMA, PS, and PMMA/PS composite latex.

PMMA/PS composite polymer latex synthesized without swelling is shown as Figure 1d, the same as in Figure 1c. The PMMA seed latex was linear structure, and thus the styrene monomer diffused into the seed latex easily during polymerization and the reaction proceeded in the PMMA seed latex to form the morphology that PS dispersed in the PMMA zone. AIBN was used as initiator in the second stage to synthesize the PMMA/PS composite polymer latex with homogeneous morphology, as shown as Figure 1e. AIBN was oil-soluble, and dissolved in the styrene monomer and swelled into the PMMA seed latex completely during swelling. So that the second stage reaction proceeded in the inner region of PMMA seed latex to form the morphology with PS distributed homogeneously in the PMMA region. The morphology of the PMMA/PS composite polymer latex, synthesized without swelling is shown as Figure 1f the same as in Figure 1e. The styrene monomer easily diffused into the linear PMMA seed latex and polymerized in the inner region of PMMA seed latex to form a homogeneous morphology.

\section{TLC Analysis}

Qualitative Analysis. Figure 2 shows the results of TLC analysis of PMMA, PS and PMMA/PS composite polymer (MS-KPS) obtained from emulsion latexes. Pure PMMA stayed at the original point, and pure PS moved over $9 \mathrm{~cm}$ from the original place. The PMMA/PS composite polymer latex decomposed into three components, at the original place, $6 \mathrm{~cm}$ from the original place, and $9 \mathrm{~cm}$ from the original place. This indicates that a thin layer of the PMMA-graft-PS copolymer forms between the PS and PMMA regions of the PMMA/PS composite polymer latex. Other composite polymer latexes, such as MS-ABCVA and MS-AIBN analyzed by TLC had the same results as MS-KPS.
Table II. Weight percentage of PMMA-graft-PS copolymer

\begin{tabular}{cc}
\hline PMMA/PS compositelatex & $\begin{array}{c}\text { Weight percentage of PMMA-graft-PS } \\
\text { copolymer }\end{array}$ \\
\hline MS-KPS & $5.2 \%$ \\
MS-ABCVA & $6.8 \%$ \\
MS-AIBN & $6.9 \%$ \\
\hline
\end{tabular}

Quantitative Analysis. Table II shows the results of TLC-FID analysis for the PMMA/PS composite polymer obtained from the emulsion latex. The weight percentage of PMMA-graft-PS copolymer of MS-AIBN approached that of MS-ABCVA, but the weight percentage of PMMA-graft-PS copolymer of MS-KPS was less than that of MS-ABCVA and MS-AIBN. Since the reaction zone of the second stage reaction for the synthesis of MSABCVA and MS-AIBN proceeded throughout the inner region of seed latex particles, but only on the shell region of seed latex particles for the synthesis of MS-KPS. The reaction zone of the former was larger than the later. The weight percentage of PMMA-graft-PS copolymer of MS-KPS was thus less than that of MS-ABCVA and MSAIBN.

Compatibility of the Components of PMMA/PS Composite Polymer

Table III shows the compatibility of PMMA and PS components. Compatibility of PMMA/PS composite polymer latex was better than that of conventional PMMA/ PS blends. The molecular weights of MS-KPS, MSABCVA, MS-AIBN, and commercial PMMA/PS polymer blend were approximately, shown in Table I, and thus the effects of molecular weight on the compatibility of the components of PMMA/PS composite polymer were neglected. But the interpenetration layer in the PMMA/ PS composite polymer latex influenced the compatibility of the components significantly. The interpenetration layer contained PMMA-graft-PS copolymer ${ }^{15}$ and PMMA, PS polymer chains entanglement, ${ }^{16}$ both of which increased the compatibility of PMMA and PS components. The compatibility of components of MS-ABCVA and MS-AIBN was larger than that of MS-KPS. Owing to the amount of PMMA-graft-PS copolymer, which increased the compatibility of PMMA and PS phases. According to TLC-FID analysis, the amount of PMMAgraft-PS copolymer of MS-ABCVA and MS-AIBN was larger than that of MS-KPS. So the compatibility of the components of MS-ABCVA and MS-AIBN was better than that of MS-KPS.

\section{Effects of Thermal Annealing on the Morphology of PMMA/PS Composite Poylmer}

Figures $3 a-3 d$ show TEM photographs of effects of thermal annealing on the morphology of conventional PMMA and PS polymer blend. After the polymer blend was annealed, the domain size of PMMA phase increased to reduce the interfacial area and interfacial tension. Figures $4 \mathrm{a}-4 \mathrm{~d}$ show TEM photographs of the effects of thermal annealing on the morphology of PMMA/PS composite polymer obtained from MS-KPS. The morphology of MS-KPS was a core--shell. The core was PMMA and shell was PS. After thermal annealing, the core and shell were disrupted, and the PMMA and 
Table III. Glass transition temperature $\left(T_{\mathrm{g}}\right)$ of PMMA, PS, and PMMA/PS composite polymer latexes

\begin{tabular}{lcccc}
\hline & $\begin{array}{c}T_{\mathrm{g}} \text { of PMMA seed } \\
\left(T_{\mathrm{g} \text { PMMA }}^{0}\right.\end{array}$ & $\begin{array}{c}T_{\mathrm{g}} \text { of PS } \\
\left(T_{\mathrm{g} \text { PS }}^{0}\right)\end{array}$ & $\begin{array}{c}T_{\mathrm{g}} \text { of PMMA/PS } \\
\text { composite polymer latex } \\
\left(T_{\mathrm{g} \text { PMA }}\right)\end{array}$ & $\begin{array}{c}\text { Degree of compatibility of } \\
\text { PMMA and PS phase } \\
\left(T_{\mathrm{gPS}}\right)\end{array}$ \\
\hline MS-KPS & 126.96 & 96.26 & 125.84 & 104.02 \\
\hline MS-ABCVA & 126.96 & 99.65 & 119.16 & 104.6 \\
\hline MS-AIBN & 126.96 & 95.21 & 103.67 & 99.42 \\
\hline $\begin{array}{l}\text { Conventional } \\
\text { PMMA/PS blend }\end{array}$ & 104 & 92.55 & 0.47 & 0.45 \\
\hline
\end{tabular}
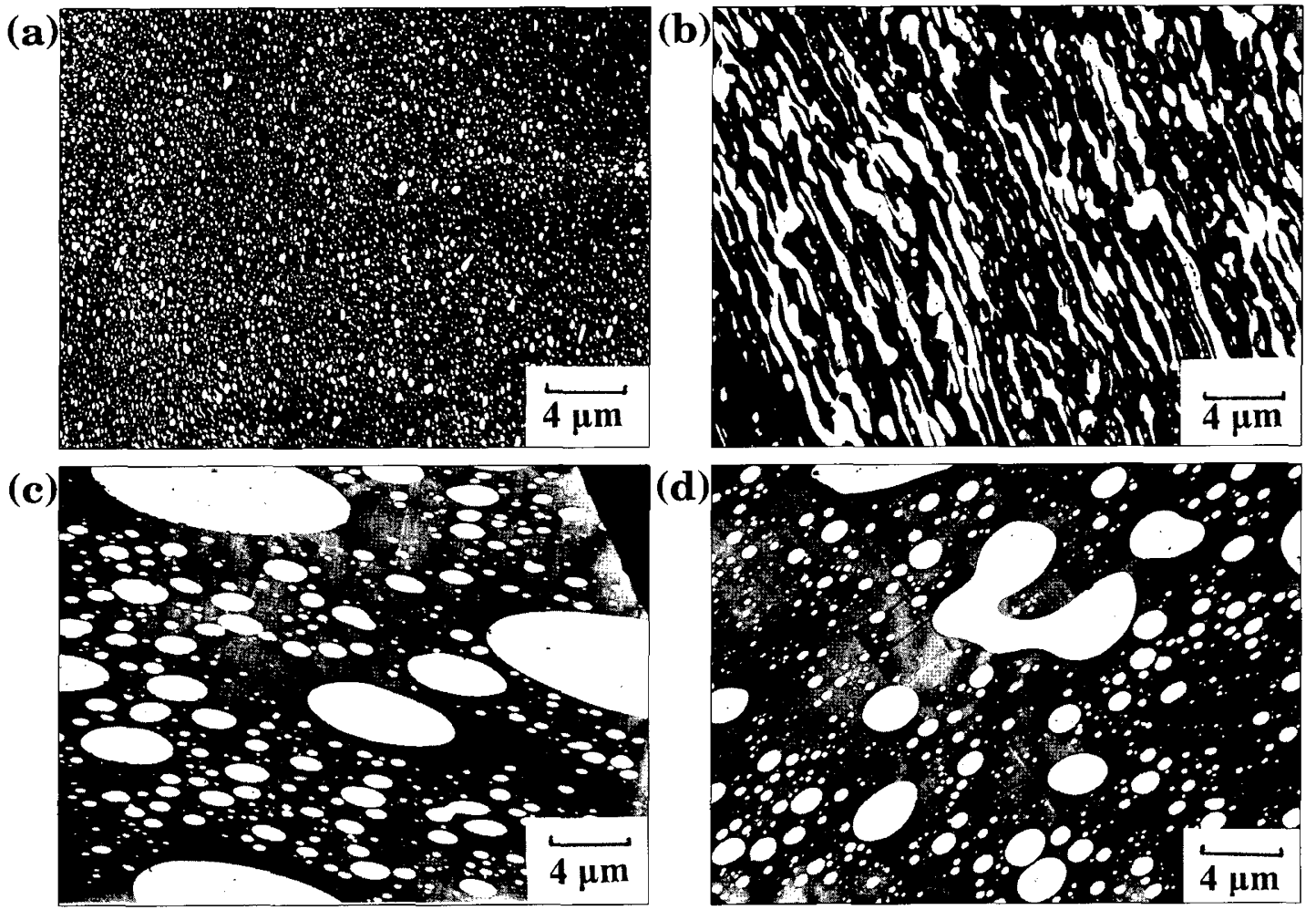

Figure 3. TEM photograph of stained cross section of the conventional PMMA/PS blend thermally annealed under $180^{\circ} \mathrm{C}($ dark $z o n e$ is PS, bright zone is PMMA). (a)Thermal annealing for 0 min. (b)Thermal annealing for 30 min. (c)Thermal annealing for 90 min. (d)Thermal annealing for $120 \mathrm{~min}$.

PS phases interpenetrated with annealing time. After the composite polymer was annealed for $90 \mathrm{~min}$, the interpenetration of PMMA and PS phases was significant as seen in Figure $4 \mathrm{~b}$. The morphology of composite polymer showed a co-continuous structure, i.e., PMMA domains dispersed in the PS continuous phase and PS domains dispersed in the PMMA continuous phase. The influence of thermal annealing on the morphology of PMMA/PS composite polymer obtained from MSABCVA is shown in Figures $5 \mathrm{a}-5 \mathrm{~d}$. Throughout thermal annealing, the co-continuous structure appeared in the polymer blend, but interpenetration was not found. Because compatibility of the components of MS-ABCVA was better than that of MS-KPS. So, under the effect of thermal annealing, the co-continuous structure was easy to form. The interpenetration structure may appear early during thermal annealing. Figures $6 a-6 d$ show the influence of thermal annealing on the morphology of composite polymer obtained from MS-AIBN, as also for MS-ABCVA, because the compatibility of the components of MS-AIBN approached that of MS-ABCVA. The effect of thermal annealing on the morphology of MS-
ABCVA was the same as that on the morphology of MSAIBN.

Effects of Thermal Annealing on the Morphology of Commercial Polymer/Composite Latex Polymer Blend

Figures $7 \mathrm{a}-7 \mathrm{~d}$ show the influence of thermal annealing on the morphology of commercial polymer/MS-KPS blend. PMMA was the dispersed phase and PS was the continuous phase. The effects of thermal annealing on the average areas of PMMA domains are shown in Table IV. The influence of thermal annealing on the domain size of PMMA disperse phase was thus insignificant, because the PMMA-graft-PS copolymer played the role of compatibilizing agent to lower interfacial tension between PMMA and PS phases of the polymer blend. The effects of thermal annealing on the morphology of commercial polymer/MS-ABCVA polymer blend and commercial polymer/MS-AIBN blend polymer are shown in Figures $8 \mathrm{a}-8 \mathrm{~d}$ and Figures $9 \mathrm{a}-9 \mathrm{~d}$ respectively. The morphologies of the two polymer blends were the same as commercial polymer/MS-KPS polymer blend. Effects of thermal annealing on the domain size of PMMA dis- 
(a)

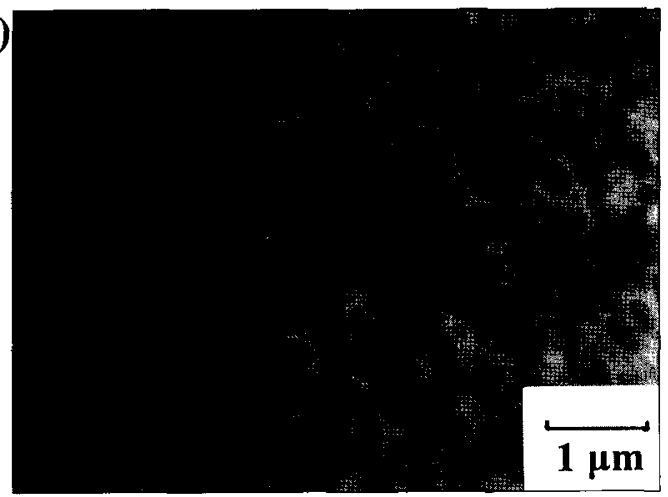

(c)

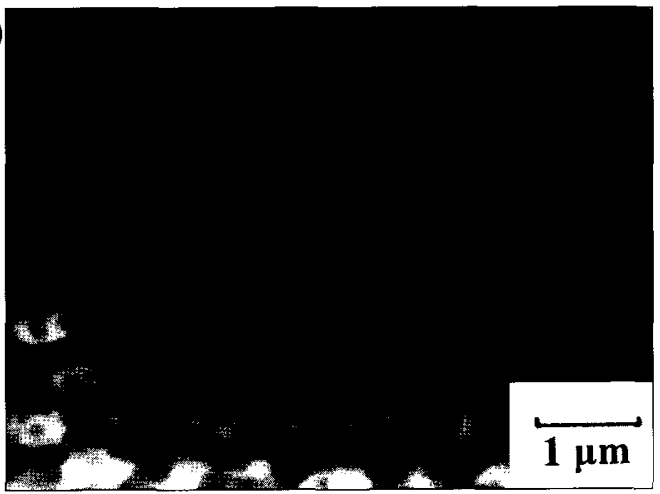

(b)

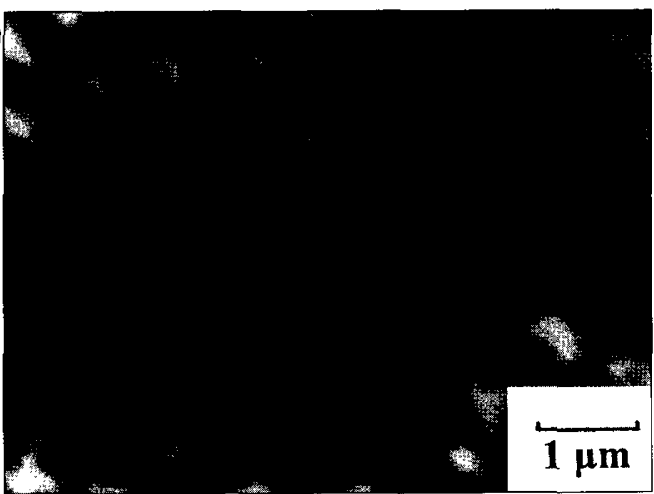

(d)

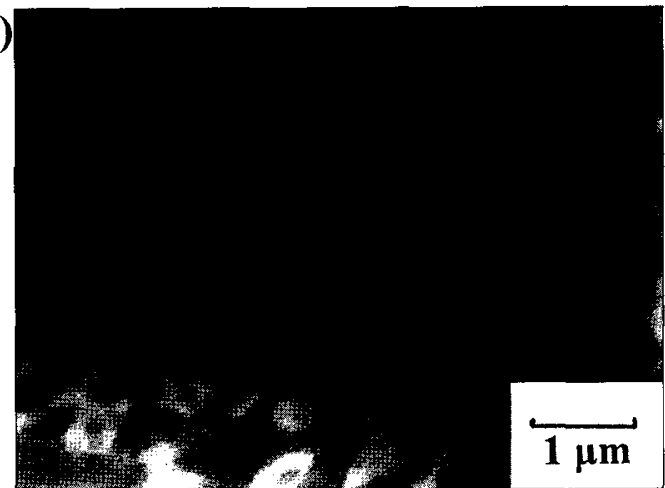

Figure 4. TEM photograph of the stained cross section of the composite polymer obtained from MS-KPS thermally annealed under $180^{\circ} \mathrm{C}$ (dark zone is PS, bright zone is PMMA). (a)Thermal annealing for $30 \mathrm{~min}$. (b)Thermal annealing for $90 \mathrm{~min}$. (c)Thermal annealing for 150 min. (d)Thermal annealing for $180 \mathrm{~min}$.

(a)

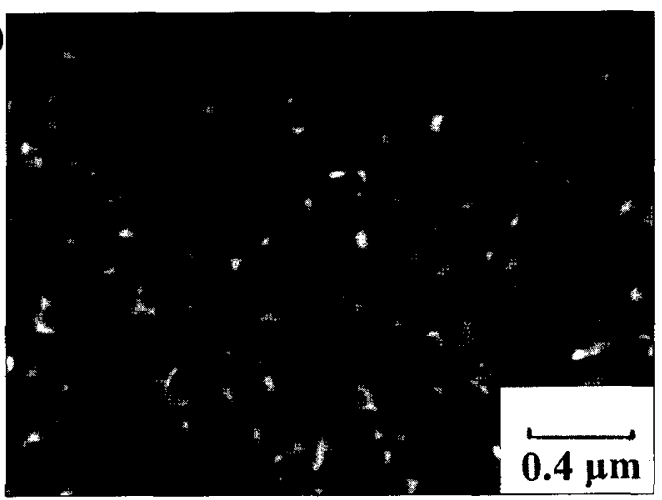

(c)

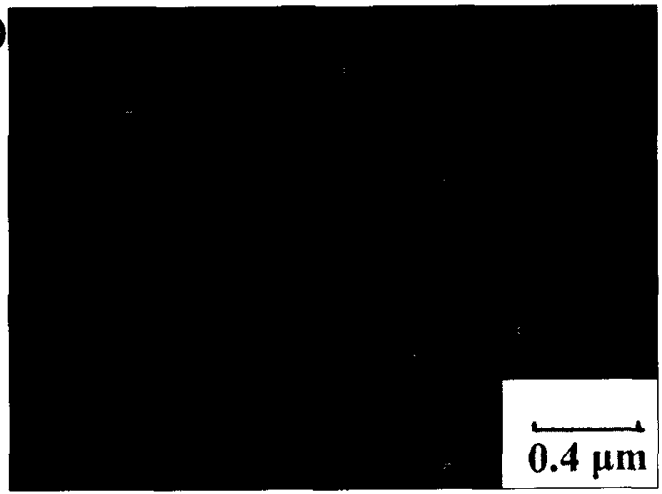

(b)

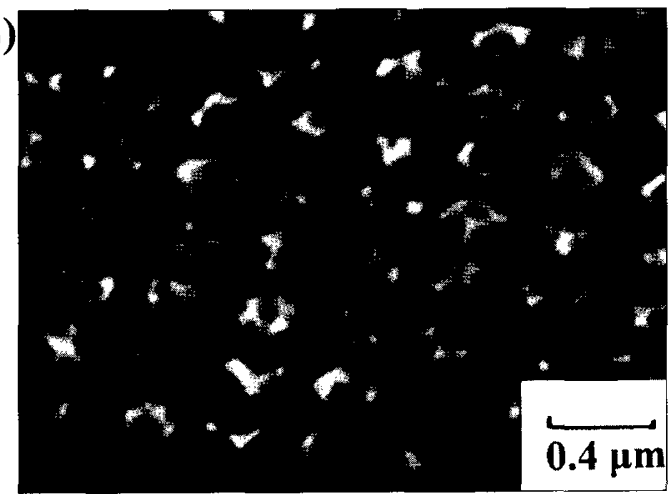

(d)

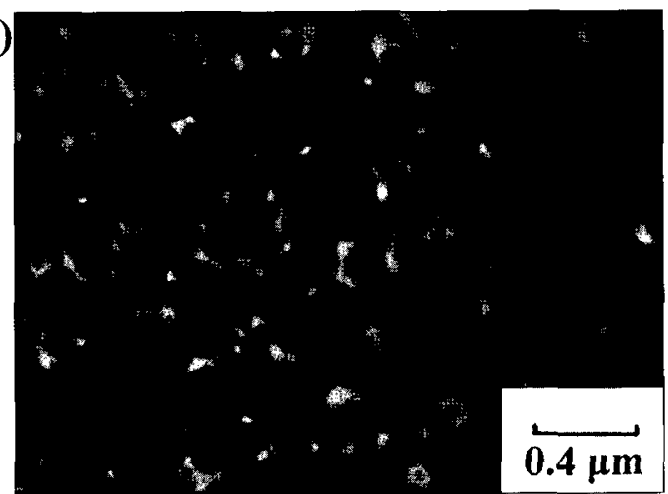

Figure 5. TEM photograph of the stained cross section of the composite polymer obtained from MS-ABCVA thermally annealed under $180^{\circ} \mathrm{C}$ (dark zone is PS, bright zone is PMMA). (a)Thermal annealing for $30 \mathrm{~min}$. (b)Thermal annealing for $90 \mathrm{~min}$. (c)Thermal annealing for 120 min. (d)Thermal annealing for $180 \mathrm{~min}$. 
(a)

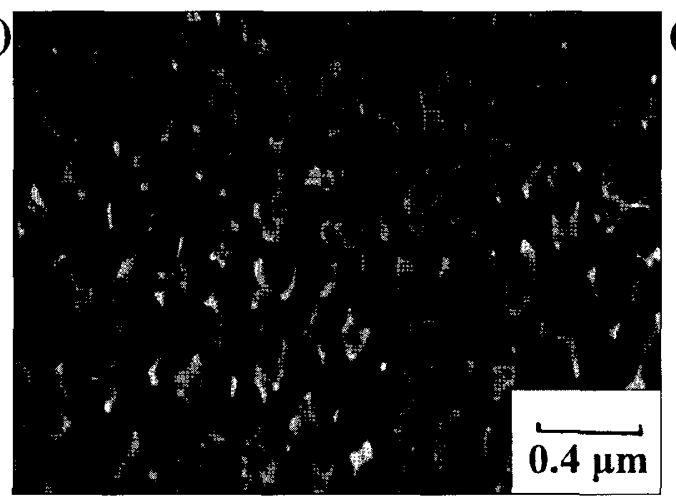

(c)

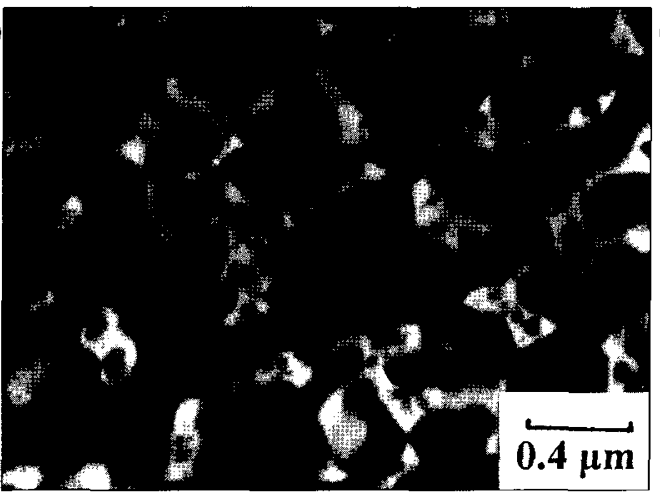

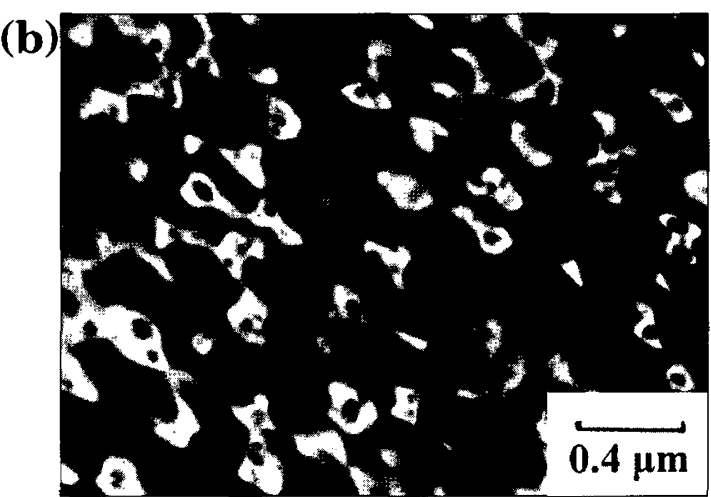

(d)

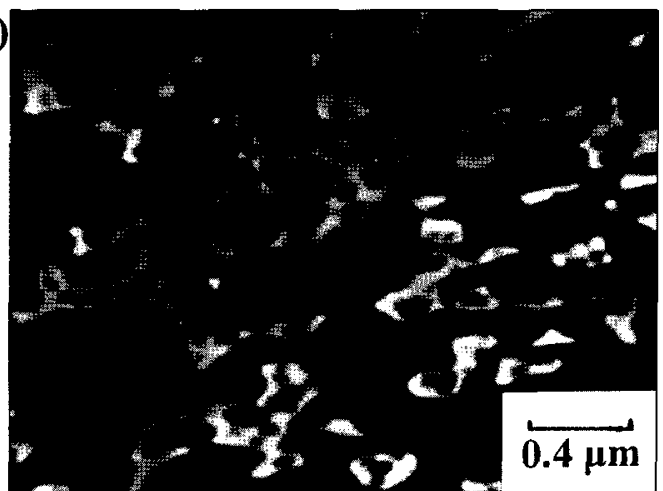

Figure 6. TEM photograph of the stained cross section of the composite polymer obtained from MS-AIBN thermally annealed under $180^{\circ} \mathrm{C}$ (dark zone is PS, bright zone is PMMA). (a)Thermal annealing for $30 \mathrm{~min}$. (b)Thermal annealing for 90 min. (c)Thermal annealing for 120 min. (d)Thermal annealing for $180 \mathrm{~min}$.
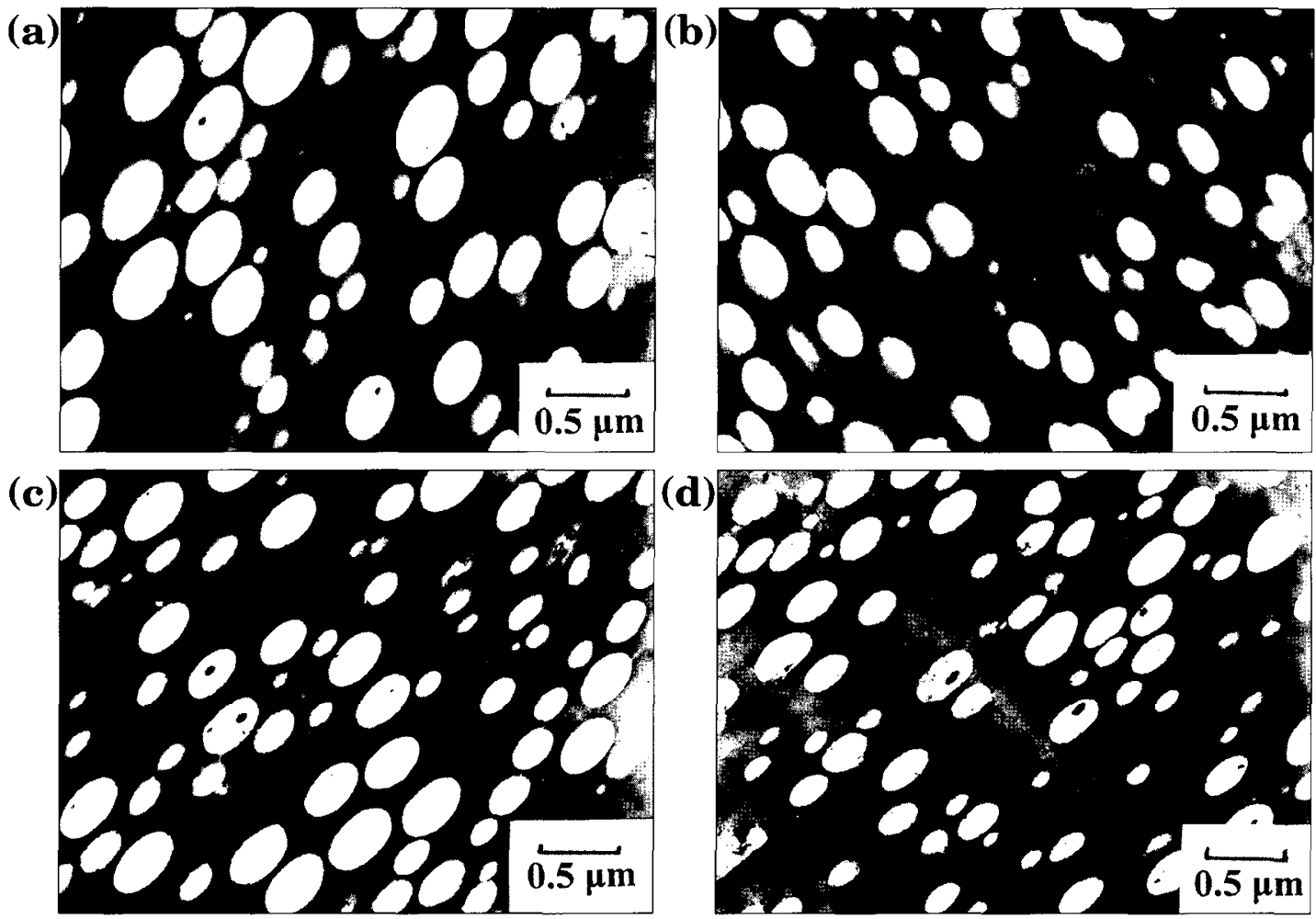

Figure 7. TEM photograph of the stained cross section of the commercial polymer/MS-KPS blend thermally annealed under $180^{\circ} \mathrm{C}(\mathrm{dark}$ zone is PS, bright zone is PMMA). (a)Thermal annealing for 0 min. (b)Thermal annealing for $60 \mathrm{~min}$. (c)Thermal annealing for $150 \mathrm{~min}$. (d)Thermal annealing for $180 \mathrm{~min}$. 
(a)

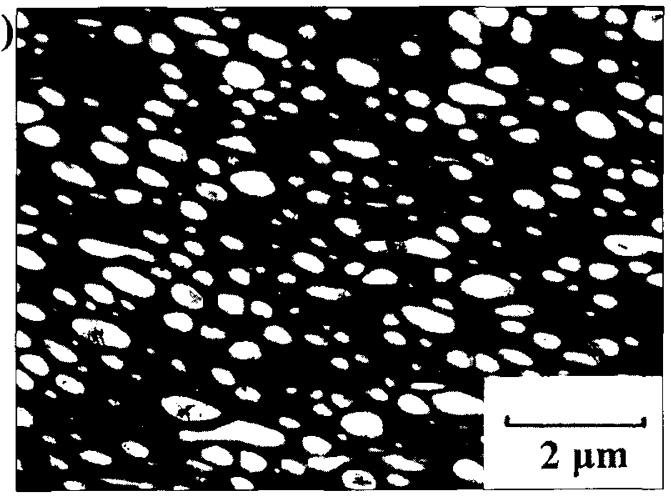

(c)

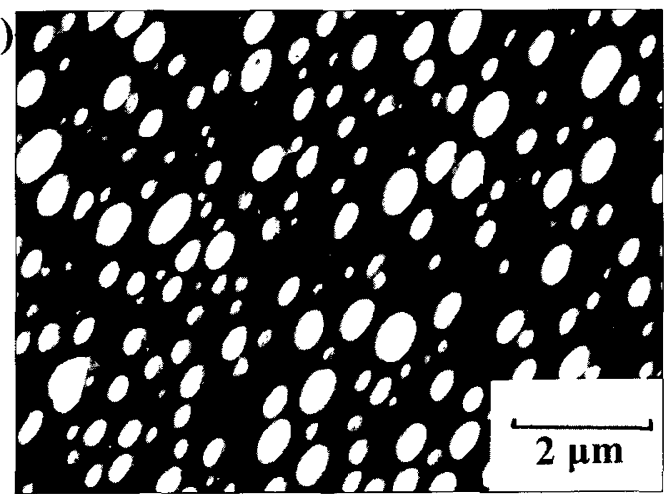

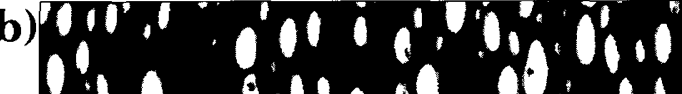

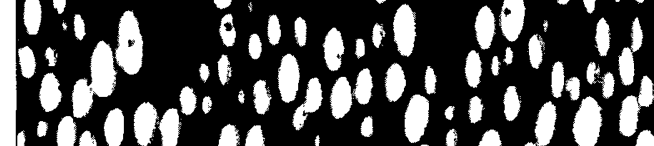

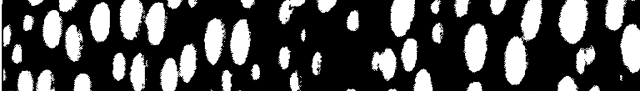

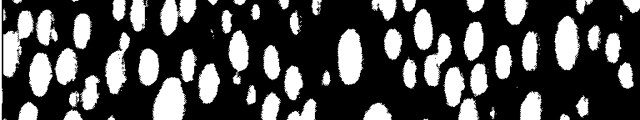

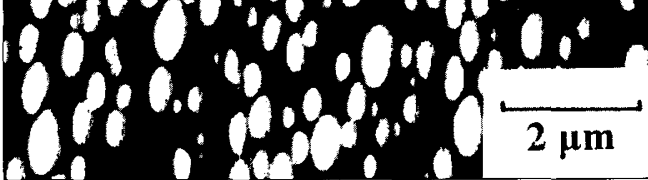

(d)

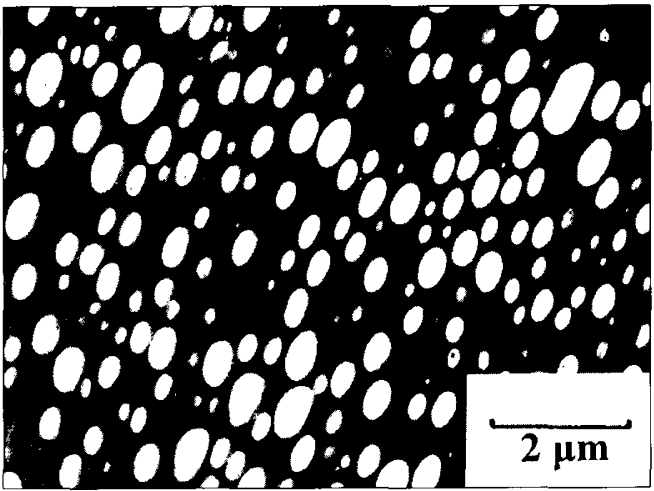

Figure 8. TEM photograph of the stained cross section of the commercial polymer/MS-ABCVA blend thermally annealed under $180^{\circ} \mathrm{C}$ (dark zone is PS, bright zone is PMMA). (a)Thermal annealing for 0 min. (b)Thermal annealing for 60 min. (c)Thermal annealing for 120 min. (d)Thermal annealing for $180 \mathrm{~min}$.

(a)

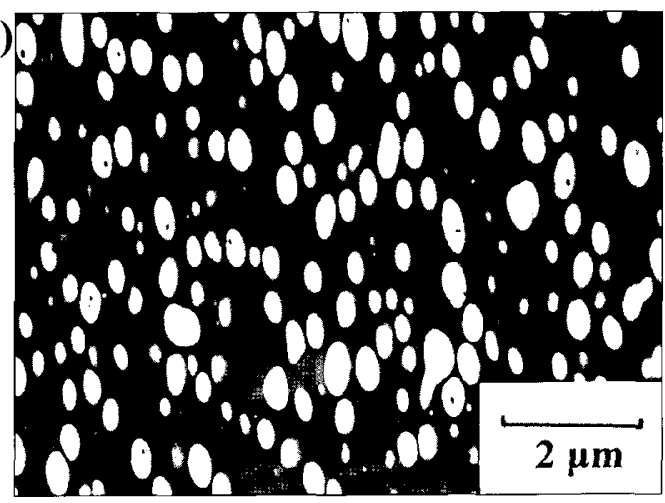

(c)

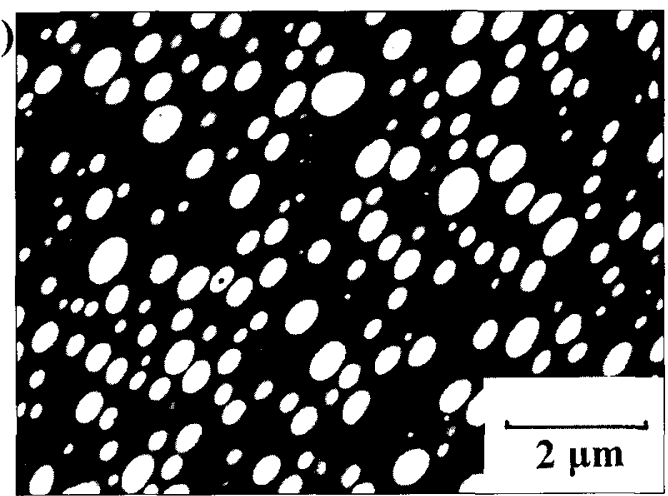

(b)

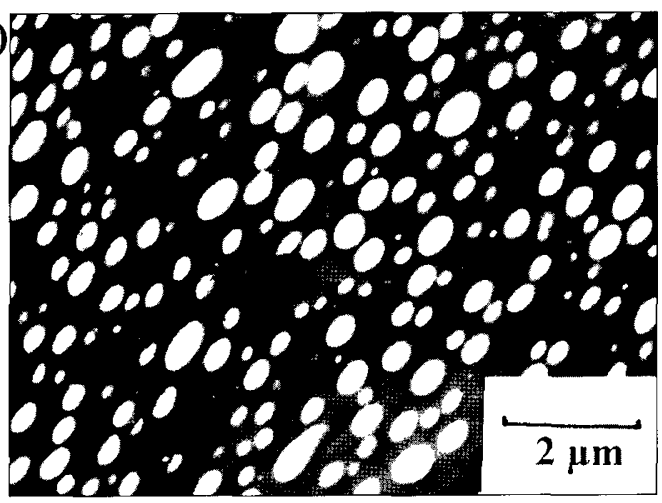

(d)

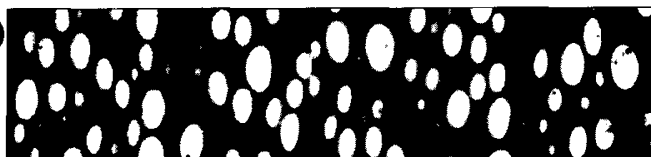
60.80009000

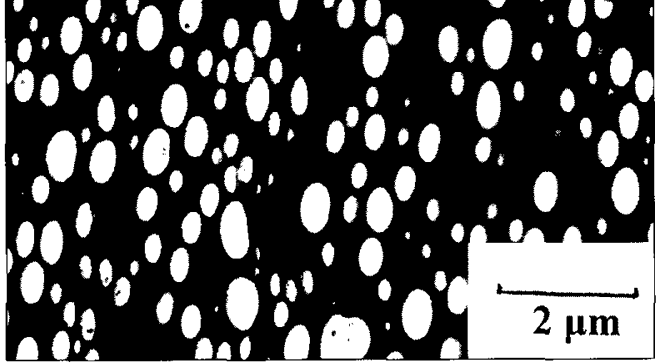

Figure 9. TEM photograph of the stained cross section of the commercial polymer/MS-AIBN blend thermally annealed under $180^{\circ} \mathrm{C}($ dark zone is PS, bright zone is PMMA). (a)Thermal annealing for $0 \mathrm{~min}$. (b)Thermal annealing for $60 \mathrm{~min}$. (c)Thermal annealing for $120 \mathrm{~min}$. (d)Thermal annealing for $180 \mathrm{~min}$. 
Table IV. Effects of thermal annealing on the domain size of PMMA dispersed phase of polymer blends

\begin{tabular}{lrc}
\hline & Annealing time & \multicolumn{2}{c}{$\begin{array}{c}\text { Average domain size of } \\
\text { PMMA dispersed phase }\end{array}$} \\
\cline { 2 - 2 } Polymer blend & min & $\mathrm{nm}^{2}$ \\
\hline & 60 & 95897 \\
Commercial & 150 & 96203 \\
polymer/MS-KPS & 180 & 96193 \\
& 0 & 96326 \\
\hline & 60 & 78986 \\
Commercial & 120 & 79232 \\
polymer/MS-ABCVA & 180 & 79872 \\
& 0 & 79986 \\
\hline & 60 & 79850 \\
Commercial & 120 & 80363 \\
polymer/MS-AIBN & 180 & 79614 \\
& & 80722 \\
\hline
\end{tabular}

persed phases were insignificant as seen in Table IV. But the average area of PMMA dispersed phase of commercial polymer/MS-KPS polymer blend was larger than that of commercial polymer/MS-ABCVA polymer blend and commercial polymer/MS-AIBN polymer blend as shown in Table IV. According to TLC-FID analysis, the amount of PMMA-graft-PS copolymer of MS-KPS was less than that of MS-ABCVA and MS-AIBN. So, the compatibility of the components of commercial polymer/MSKPS blend was poorer than that of commercial polymer/ MS-ABCVA blend and commercial polymer/MS-AIBN blend. The domain size of PMMA disperse phase of commercial polymer/MS-KPS blend was larger than that of commercial polymer/MS-ABCVA polymer blend and commercial polymer/MS-AIBN polymer blend.

\section{CONCLUSION}

The PMMA/PS composite polymer latex was synthesized by soapless seeded emulsion polymerization. The effect of initiator of second stage reaction on the morphology of composite polymer latex was significant. The morphology of MS-KPS was a core-shell. The morphology of MS-ABCVA shows core and shell regions. The core region was PS, and the shell region showing PS was dispersed in the PMMA continuous phase. The morphol- ogy of MS-AIBN was homogeneous. The PMMA/PS composite polymer latex contained the PMMA-graft-PS copolymer, which increased the compatibility of the components of composite polymer latex. Increase of the amount of PMMA-graft-PS copolymer increased the compatibility of the PMMA/PS composite polymer latex. The compatibility of the components of MS-AIBN and MS-ABCVA was larger than that of MS-KPS. Under thermal annealing, the morphology of composite polymer showed the co-continuous structure due to the PMMA-graft-PS copolymer that increased the compatibility of composite polymer. Under thermal annealing, the PMMA-graft-PS copolymer stabilized the domain size of the PMMA dispersed phase of commercial polymer/composite latex polymer blend.

\section{REFERENCE}

1. J. L. Jonsson, H. Hassande, L. H. Jonsson, and B. Tornell, Macromolecules, 24, 126 (1991).

2. T. I. Min, A. Klein, M. S. El-Aasser, and J. W. Vanderhoff, $J$. Polym. Sci. Polym. Chem. Ed., 21, 2845 (1983).

3. D. I. Lee and T. Ishikawa, J. Poly. Sci. Polym. Chem. Ed., 21, 147 (1983).

4. R. A. Dickie, M. F. Cheung, and S. Newman, J. Appl. Polym. Sci., 17, 65 (1973).

5. D. J. Hourston, R. Satgurunthan, and H. Varma, J. Appl. Polym. Sci., 31, 1955 (1986).

6. I. Cho and K. W. Lee, J. Appl. Polym. Sci., 30, 1903 (1985).

7. M. Okubo, Y. Katsuta, and T. Matsumoto, J. Polym. Sci. Polym. Lett. Ed., 18, 481 (1980).

8. M. Okubo, M. Ando, A. Yamada, Y. Katsuta, and T. Matsumoto, J. Polym. Sci. Polym. Lett. Ed., 19, 143 (1981).

9. M. Okubo, A. Yamada, Y. Katsuta, and T. Matsumoto, J. Polym. Sci. Polym. Lett. Ed., 16, 3219 (1980).

10. S. Muroi and K. Hosoi, J. Polym. Sci. Polym. Chem. Ed., 22, 1365 (1984).

11. C. F. Lee and K. R. Lin, and W. Y. Chiu, J. Appl. Polym. Sci., 51, 1621 (1994).

12. C. F. Lee and W. Y. Chiu, J. Appl. Polym. Sci., 56, 1263 (1995).

13. C. F. Lee, W. Y. Chiu, and Y. C. Chern, J. Appl. Polym. Sci., 57, 591 (1995).

14. C. F. Lee and W. Y. Chiu . J. Appl. Polym. Sci., 65, 425 (1997).

15. C.F. Lee, Y. H. Chen, and W. Y. Chiu, J Appl. Polym. Sci., 69, 13 (1998).

16. C. F. Lee, Polymer, 41, 1337 (2000).

17. L. H. Chu, Ph.D. Thesis, National Taiwan University, 1994. 